\title{
The impact of COVID-19 on cardiology departments
}

\author{
Kamleshun Ramphul ${ }^{1}$, Petras Lohana ${ }^{2}$, Renuka Verma ${ }^{3}$, Shaheen Sombans ${ }^{4}$
}

${ }^{1}$ Department of Pediatrics, Shanghai Xin Hua Hospital, School of Medicine, Shanghai Jiao Tong University, Shanghai, China

2Department of Internal Medicine, Liaquat University of Medical and Health Sciences Hospital, Jamshoro, Pakistan

${ }^{3}$ Department of Internal Medicine, Guru Gobind Singh Medical College, Punjab, India ${ }^{4}$ Department of Internal Medicine and Cardiology, Bharati Vidyapeeth University

Medical College and Hospital, Pune, India

Submitted: 11 February 2021

Accepted: 21 March 2021

Arch Med Sci Atheroscler Dis 2021; 6: e1-e2

DOI: https://doi.org/10.5114/amsad.2021.105063

Copyright @ 2021 Termedia \& Banach

The spread of the Coronavirus disease 2019 (COVID-19) pandemic throughout the world has persisted for over a year now [1]. Multiple hospitals in several countries do not have any free beds available and the health care providers are facing burn-out and fatigue. Some hospital centres such as in Lombardy, Italy even became an epicentre of infections [2]. We sought to briefly describe the changes seen and reported in literature by several cardiology departments from various regions across the world.

Davis et al. provided multiple guidance tips to maximize the care of patients with a history of cardiovascular disease while limiting the risk towards health care providers [3]. They suggested that hospital care for patients with no positive symptoms or tests of COVID-19 should still follow protocols to limit face-to-face contact. Timings and priorities of non-emergency procedures should also be properly reassessed. Out-patient care should be continued as closely as possible so that patients have proper compliance and access to their medications. To adhere with several protocols, the use of angiotensin converting enzyme inhibitors (ACEi) and angiotensin II receptor blockers (ARBs) has also been recommended by many cardiologists despite possible pathophysiological roles of COVID-19 [4-6]. It is also vital to properly differentiate COVID-19 symptoms from symptoms of cardiovascular diseases. Chang et al. and Guragai et al. both reported cases of previously unrecognized Brugada syndrome in COVID-19 patients presenting with fever [7, 8]. Close monitoring of COVID-19 patients has also been highly encouraged because they are prone to several cardiovascular complications such as arrythmias, myocardial infarction, and stroke. The use of antiviral therapy drugs with a pro-arrhythmic risk should also be carefully discussed and the risks and benefits evaluated [3]. With the rise in digital revolution and growing presence in healthcare, Leite-Moreira et al. also discussed the use of several such gadgets to help protect and improve the outcome of patients with a past cardiovascular history. These apps and services can educate the at-risk groups, as well as track their progress and provide timely help as required [9]. Because cardiologists are often required to monitor COVID-19 patients in the ICUs, they are equally at risk of contracting the virus. Proper personal protective equipment (PPE) and vaccinations should be advised for all cardiologists, and regular testing and health protocols should be closely followed [10].
Corresponding author:

Dr. Shaheen Sombans

Bharati Vidyapeeth

University Medical

College and Hospital

Pune, India

Medical College Road

Pune - Satara Rd

Dhankawadi, Pune

Maharashtra 411043

India

Phone: +91 9373728706

E-mail: drshaheensombans@ gmail.com 
Previous data have showed that cardiologists suffer from one of the highest burn-out rates and longest working hours among physicians. However, only $23 \%$ of cardiologists are willing to seek help when they are depressed or suicidal [11]. In their most recent article in Heart, by the British Medical Journal (BMJ), Patel et al. emphasized several methods that can be helpful to protect the mental health of cardiologists during COVID-19 [11]. Primary prevention includes preparation of healthcare workers in high-pressure environments. Proper supply of PPE, prevention measures, and methods to reduce the risk of getting COVID-19 should be encouraged. Secondary prevention should focus on protecting at-risk health care providers who have a past history of anxiety depression or any other medical illness. Proper roles should be assigned considering the stress and impact the hours of duty will have on such health care providers. In their analysis for tertiary prevention, they advised that rapid and accessible services cater for any mental health symptoms. The concept of "Proximity, Immediacy, Expectancy, and Simplicity" has also been mooted. The local psychiatric unit can try to collaborate with the affected staff to provide a safe space for them to gain access to fast and easy care [11]. Medical workers should also work with the general public to reduce the stigma of mental illness among health care providers.

Finally, the impact of COVID-19 on cardiology training was also discussed at different levels. To reduce the risk of spreading COVID-19, several medical programs have shifted to host their conferences and educational training classes online. However, there have also been many cancellations of major teaching events, and this has led to lower teaching and learning opportunities during 2020 and possibly 2021. Goel et al. also mentioned that multiple cardiology research programs have suspended or cancelled their projects or due to the inability to have proper in-person participant visits. Several grants could be lost due to the impact of the pandemic [12]. In the United States, several cardiology fellowship programs have also used a virtual online platform to conduct their interviews. While this provides a convenient and less costly approach for the applicant, it is yet to be seen if this provides them with a realistic idea of the program $[3,13,14]$.

As challenges unfold during these unprecedented and unpredictable times, many of the steps previously described should be encouraged across several countries to provide more friendly care for those who are putting themselves at risk to treat COVID-19 patients, and hopefully "this too shall pass" [12].

\section{Conflict of interest}

The authors declare no conflict of interest.

\section{References}

1. Ramphul K, Mejias SG. Coronavirus disease: a review of a new threat to public health. Cureus 2020; 12: e7276.

2. Remuzzi A, Remuzzi G. COVID-19 and Italy: what next? Lancet 2020; 395: 1225-8.

3. Davis GK, Adlan A, Majewski J, Ibrahim B. SARS-CoV-2 pandemic and the cardiovascular system: what the non-cardiologist needs to know. Clin Med 2020; 20: 262-5.

4. Mehta N, Kalra A, Nowacki AS, et al. Association of use of angiotensin-converting enzyme inhibitors and angiotensin II receptor blockers with testing positive for coronavirus disease 2019 (COVID-19). JAMA Cardiol 2020; 5: 1020-6.

5. Kuster GM, Pfister O, Burkard T, et al. SARS-CoV2 should inhibitors of the renin-angiotensin system be withdrawn in patients with COVID-19? Eur Heart J 2020 41: 1801-3.

6. Fosbøl EL, Butt JH, Østergaard L, et al. Association of angiotensin-converting enzyme inhibitor or angiotensin receptor blocker use with COVID-19 diagnosis and mortality. JAMA 2020; 324: 168-77.

7. Chang D, Saleh M, Garcia-Bengo Y, Choi E, Epstein L, Willner J. COVID-19 infection unmasking brugada syndrome. HeartRhythm Case Rep 2020; 6: 237-40.

8. Guragai N, Posimreddy S, Vasudev R, et al. Unmasking of Brugada syndrome in a patient with Coronavirus disease 2019 (COVID-19). Acta Cardiol 2020: https://doi.or g/10.1080/00015385.2020.1761583.

9. Leite-Moreira AF, Adão R. Mobile cardiology during the COVID-19 outbreak. Cardiovasc Res 2020; 116: e149-51.

10. Monsuez JJ. Cardiology practice in the COVID-19 era. Eur J Prev Cardiol 2020; 27: 1133-5.

11. Patel RK, Sweeney MD, Baker CSR, et al. If not now, when? Enhancing cardiologists' psychological well-being as a COVID-19 gain. Heart 2021; 107: 593-5.

12. Goel S, Sharma A. COVID-19 pandemic and its impact on cardiology and its subspecialty training. Progress Cardiovasc Dis 2020; 63: 525-6.

13. Wolff $M$, Burrows $H$. Planning for virtual interviews: residency recruitment during a pandemic. Acad Pediatr 2021; 21: 24-31.

14. Jones RE, Abdelfattah KR. Virtual interviews in the era of COVID-19: a primer for applicants. J Surg Educ 2020; 77: 733-4. 